\title{
Early Morphofunctional Changes in Angll-Infused Mice Contribute to Regional Onset of Aortic Aneurysm and Dissection
}

\author{
Lydia Aslanidou ${ }^{a, b} \quad$ Bram Trachet $^{a, c}$ Linda Sasset $^{b}$ Goran Lovric ${ }^{d, e}$ \\ Nikolaos Stergiopulos ${ }^{a}$ Annarita Di Lorenzo ${ }^{b}$ \\ anstitute of Bioengineering, École Polytechnique Fédérale de Lausanne, Lausanne, Switzerland; bepartment \\ of Pathology and Laboratory Medicine, Cardiovascular Research Institute, Feil Family Brain and Mind Research \\ Institute, Weill Cornell Medicine, New York, NY, USA; 'BioMMeda, Ghent University, Ghent, Belgium; \\ dCentre d'Imagerie BioMédicale, École Polytechnique Fédérale de Lausanne, Lausanne, Switzerland; eSwiss Light \\ Source, Paul Scherrer Institute, Villigen, Switzerland
}

\section{Keywords}

Angiotensin II - Aortic aneurysm · Aortic dissection ·

Vascular reactivity $\cdot$ Synchrotron

\begin{abstract}
Aortic aneurysms and dissections are silent and lethal conditions, whose pathogenesis remains incompletely understood. Although angiotensin II (Angll)-infused ApoE-/mice have been widely used to study aortic aneurysm and dissection, early morphofunctional alterations preceding the onset of these conditions remain unknown. The goal of this study was to unveil early morphofunctional changes underlying the onset of aneurysm and dissection. At 3 days post-Angll infusion, suprarenal abdominal aorta presented significant volumetric dilatation and microstructural damage. Ex vivo assessment of vascular reactivity of the suprarenal dissection-prone aorta and its side branches, showed an endothelial and contractile dysfunctions that were severe in the suprarenal aorta, moderate distally, and absent in the side branches, mirroring the susceptibility to dissection of
\end{abstract}

karger@karger.com www.karger.com/jvr

Karger $\stackrel{\text { ' }}{=}$

GOPEN ACCESS
(C) 2020 The Author(s)

Published by S. Karger AG, Basel

This article is licensed under the Creative Commons AttributionNonCommercial-NoDerivatives 4.0 International License (CC BYNC-ND) (http://www.karger.com/Services/OpenAccessLicense) Usage and distribution for commercial purposes as well as any distribution of modified material requires written permission. these different vascular segments. Early and specific morphofunctional changes of the suprarenal aorta may contribute to the regional onset of aortic aneurysm and dissection by exacerbating the biomechanical burden arising from its side branches.

(C) 2020 The Author(s)

Published by S. Karger AG, Basel

\section{Introduction}

Aortic dissections (AD) and aortic aneurysms (AA) constitute a leading cause of morbidity and mortality in the aging Western population [1]. While the AD involves an acute disruption of the media layer, followed by the formation of a false lumen and/or intramural hematoma [2] and potential organ ischemia [3], the AA is a progressive focal dilation of the aortic wall. Both $\mathrm{AD}$ and $\mathrm{AA}$ can culminate in aortic rupture [4]. Clinically, AA manifest mainly in the thoracic (ascending) and abdominal aorta. Despite sharing similar gross characteristics, ascending and abdominal aneurysms constitute disparate diseases 
driven by different mechanisms [5, 6]. Inflammation contributes to the development of $\mathrm{AD}$, involving medial degradation mediated by inflammatory cells and predisposition to dissection [7]. The etiology of the aortopathies is still poorly understood. Most of the clinical data have been obtained by the analysis of AA or AD specimens. Therefore, animal models are particularly valuable to study the functional and molecular derangements leading to the onset of these diseases.

In mice, phenotypically distinct lesions appear in the ascending and the abdominal murine aorta $[8,9]$ with AA consistently forming in the ascending aorta [9] and $\mathrm{AD}$ occurring mainly in the suprarenal abdominal aorta $[8$, $10,11]$. Initial events preceding the onset of $A A$ and $A D$ in AngII-infused mice are not yet fully understood. In part, this is due to the complex array of effects induced by AngII, including vasoconstriction [12-14], increase in blood pressure [15], vascular inflammation, and remodeling $[16,17]$.

In a recent study, Bersi et al. [18] proposed that the suprarenal aorta is prone to dissection because it is unable to counterbalance the increased stress stemming from AngII-induced high blood pressure. This incapability arises from a local imbalance in the rate of matrix deposition-degradation, pointing toward a mechano-mediated mechanism. By using synchrotron-imaged suprarenal vessels, we have shown that early microruptures occur preferentially near the ostia of suprarenal side branches, with high incidence around the celiac and superior mesenteric arteries [19-21]. A synchrotron-based biomechanical study suggested that excessive strain at the branching sites of the suprarenal aorta may give rise to nucleating sites of dissection [22]. The strain-driven dissection and the mechano-mediated aneurysm hypotheses are potentially complementary in the onset of $\mathrm{AD}$.

In literature, it has been shown that long-term infusion of AngII impairs the voltage-dependent contractions of the thoracic aorta [23]. Dysfunctional smooth muscle cell (SMC) contractility strongly correlates with maladaptive aortic remodeling and fibrosis [24]. Moreover, restoring smooth muscle contractility protects thoracic AD [25], suggesting that preserving contractile capacity may reduce mechanical stress-induced pathological events leading to $\mathrm{AA}$ and $\mathrm{AD}$. Although these studies underline the importance of vascular contractility, they focus on later time points. It has not been investigated whether or not a compromised vascular function precedes the onset of these pathologies and contributes to the strain- and mechano-mediated hypotheses in the development of $\mathrm{AA}$ and $\mathrm{AD}$. Our study fills this gap.
Our results reveal a prevailing susceptibility of the proximal suprarenal aortic segment versus the side branches (including the celiac, superior mesenteric, and left renal arteries) to vascular damage and dysfunction. These findings suggest that at early time points following AngII infusion, the morphofunctional changes of suprarenal aorta may contribute to disease initiation.

\section{Materials and Methods}

\section{Mouse Model}

Twelve-week-old male ApoE-/- mice on a C57BL/6J background were infused with AngII (1,000 ng/ $\mathrm{kg} / \mathrm{min})$ as previously described [19], for 1, 2, or 3 days. At the end of the experiments, mice were euthanized with a ketamine/xylazine cocktail (100 and $15 \mathrm{mg} /$ $\mathrm{kg}$, respectively). All procedures were approved by the Ethical Committee of Canton Vaud, Switzerland (EC 2647.3), and performed according to the Directive 2010/63/EU of the European Parliament on the protection of animals used for scientific purposes.

\section{Micro-CT Imaging}

A contrast-enhanced non-gated micro-CT scan was performed at baseline (prior to AngII-releasing pump implantation) and after 1,2 , or 3 days of AngII infusion. Mice were injected with contrast agent (ExiTron nano 12,000; Miltenyi Biotech, Germany) via lateral caudal vein $(4 \mathrm{~mL} / \mathrm{g}$ of body weight) immediately prior to micro-CT imaging. Following anesthesia with isoflurane (1.5\%), the thorax and abdomen were scanned with a Quantum FX micro-CT scanner (Caliper Life Sciences, Waltham, MA, USA). The resulting DICOM images had an isotropic voxel size of $50 \mu \mathrm{m}$. Aortic volumes were quantified using the Mimics software (Materialise, Leuven, Belgium). The abdominal aorta was scanned from ca. $5 \mathrm{~mm}$ above the celiac artery to ca. $2.5 \mathrm{~mm}$ below the left renal artery. The body weight of animals at baseline and after 1, 2, or 3 days of AngII infusion was not statistically different.

\section{PCXTM Imaging}

Harvested aortic tissues were fixed in $4 \%$ PFA for $24 \mathrm{~h}$ and imaged at the Paul Scherrer Institute (Villigen, Switzerland) using propagation-based phase-contrast imaging [26] at an isotropic voxel size of $1.625 \mu \mathrm{m}$. The contrast agent used for the micro-CT scan penetrated the aortic wall intra vitam and was visible in PCXTM scans as previously described [20,22]. The volume of contrast agent in the abdominal aorta was quantified using the Mimics software (Materialise, Leuven, Belgium). The PCXTM imaging data were collected for the same vascular segments assessed in the wire myograph system.

\section{Vascular Reactivity Study}

ApoE-/- male mice on a C57BL/6J background at 12-14 weeks of age were purchased from The Jackson Laboratory. AngII-filled micro-osmotic Alzet 1003D pumps were left o.n. in sterile $0.9 \%$ $\mathrm{NaCl}$ solution at $37^{\circ} \mathrm{C}$, a priming step before implantation. After 3 days of AngII infusion (1,000 $\mathrm{ng} / \mathrm{kg} / \mathrm{min})$, the mice were euthanized and transcardially perfused with PBS at $40 \mathrm{~mm} \mathrm{Hg}$. The abdominal aorta was carefully cleaned in situ from surrounding fat tissue and excised. 


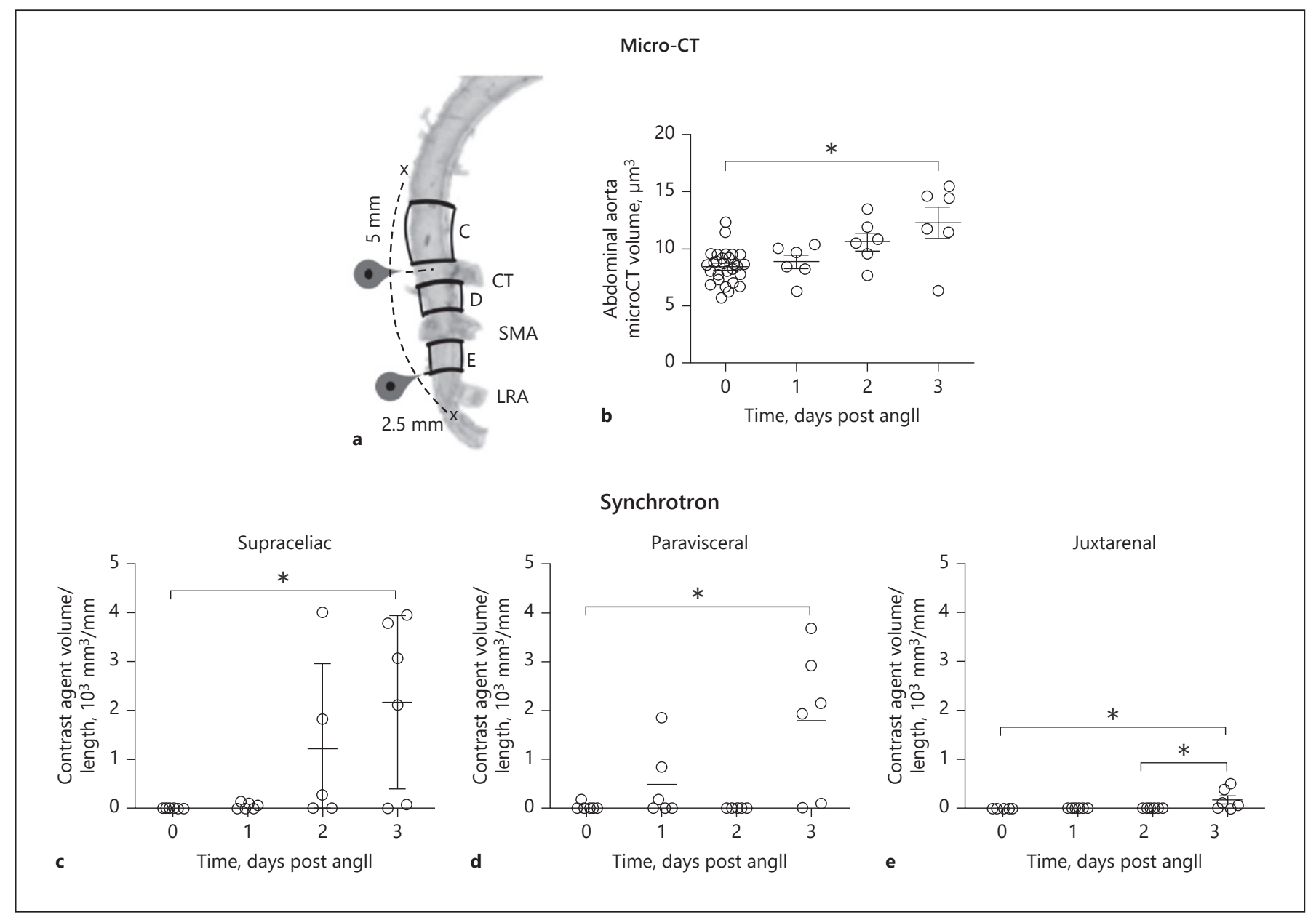

Fig. 1. In vivo micro-CT volumetric and ex vivo microvascular damage measurements of abdominal aorta segments at day 1, 2, and 3 post-AngII infusion. a Abdominal aortic volume (along the dotted line) was measured from $5 \mathrm{~mm}$ above the middle of the celiac bifurcation (shown as landmark point) to $2.5 \mathrm{~mm}$ distal to the middle of the left renal bifurcation (shown as landmark point) in micro-CT images. Local microvascular damage in synchrotron images was assessed in outlined regions 1 (supraceliac), 2 (paravisceral), and 3 (juxtarenal). b Quantification of the abdominal aortic volume in ApoE- - mice at different time points post-AngII. $n=$

The vascular functions of the following arterial segments (ca. 2 mm length) prone to $\mathrm{AA}$ and $\mathrm{AD}$ were assessed: the supraceliac segment (proximal to the celiac artery bifurcation), the paravisceral segment (between the bifurcations of the celiac and the superior mesenteric/right renal artery), and the juxtarenal segment (between the right and left renal artery). In addition, 3 aortic side branches were assessed: the celiac, superior mesenteric, and left renal arteries. The juxtarenal aortic segment and the left renal artery were considered internal controls since they are resistant to $\mathrm{AD}$ [18].

The vascular rings were mounted on a DMT620M wire myograph (Danish Myo Technology A/S, Denmark) by using a tungsten wire of $40 \mu \mathrm{m}(20 \mu \mathrm{m}$ for the celiac side branch) and carboxygenated physiological Krebs solution (118.5 mM NaCl, 25.0 mM NaH-
6 for groups of day 1, 2, and 3, $n=24$ for day 0. c-e Quantification of the contrast agent infiltrates in the abdominal aorta of ApoE-/mice following AngII infusion at the indicated times. Volume of contrast agent has been normalized to the axial length of each aortic segment and expressed as $\mu \mathrm{m}^{3} / \mu \mathrm{m} . n=5$ for supraceliac day 2; $n=6$ for the remaining groups/time points. b-e Statistical significance was assessed by Kruskal-Wallis tests among groups with Dunn's multiple comparisons. ${ }^{*} p \leq 0.05$. Data expressed as mean \pm SEM. CT, celiac trunk; SMA, superior mesenteric artery; RRA, right renal artery; LRA, left renal artery.

$\mathrm{CO}_{3}, 4.7 \mathrm{mM} \mathrm{KCl}, 1.2 \mathrm{mM} \mathrm{MgSO}_{4}, 11 \mathrm{~mm}$ glucose, and $\left.2 \mathrm{mM} \mathrm{CaCl}_{2}\right)$ at $37^{\circ} \mathrm{C}$. Vascular rings were exposed to a passive tension $(0.45 \mathrm{~g}$ for the aortic segments and superior mesenteric artery and $0.3 \mathrm{~g}$ for the celiac and left renal arteries, as determined by preliminary studies) and allowed to stabilize for at least $30 \mathrm{~min}$. Vasoconstrictions in response to phenylephrine (PE; Sigma) and TXA2 mimetic (U46619; Cayman Chem) were assessed. Vasoconstriction induced by $\mathrm{KCl}(60$ $\mathrm{mM}$ ) was used to normalize the receptor-mediated contractions. To evaluate the vasodilation to acetylcholine (ACH; Sigma) and isoproterenol (ISO; Sigma), vascular rings were preconstricted with submaximal concentrations of SER. Data were recorded by using LabChart. The length of the aortic rings was measured under a brightfield microscope (Olympus-SIS) following each experiment. 


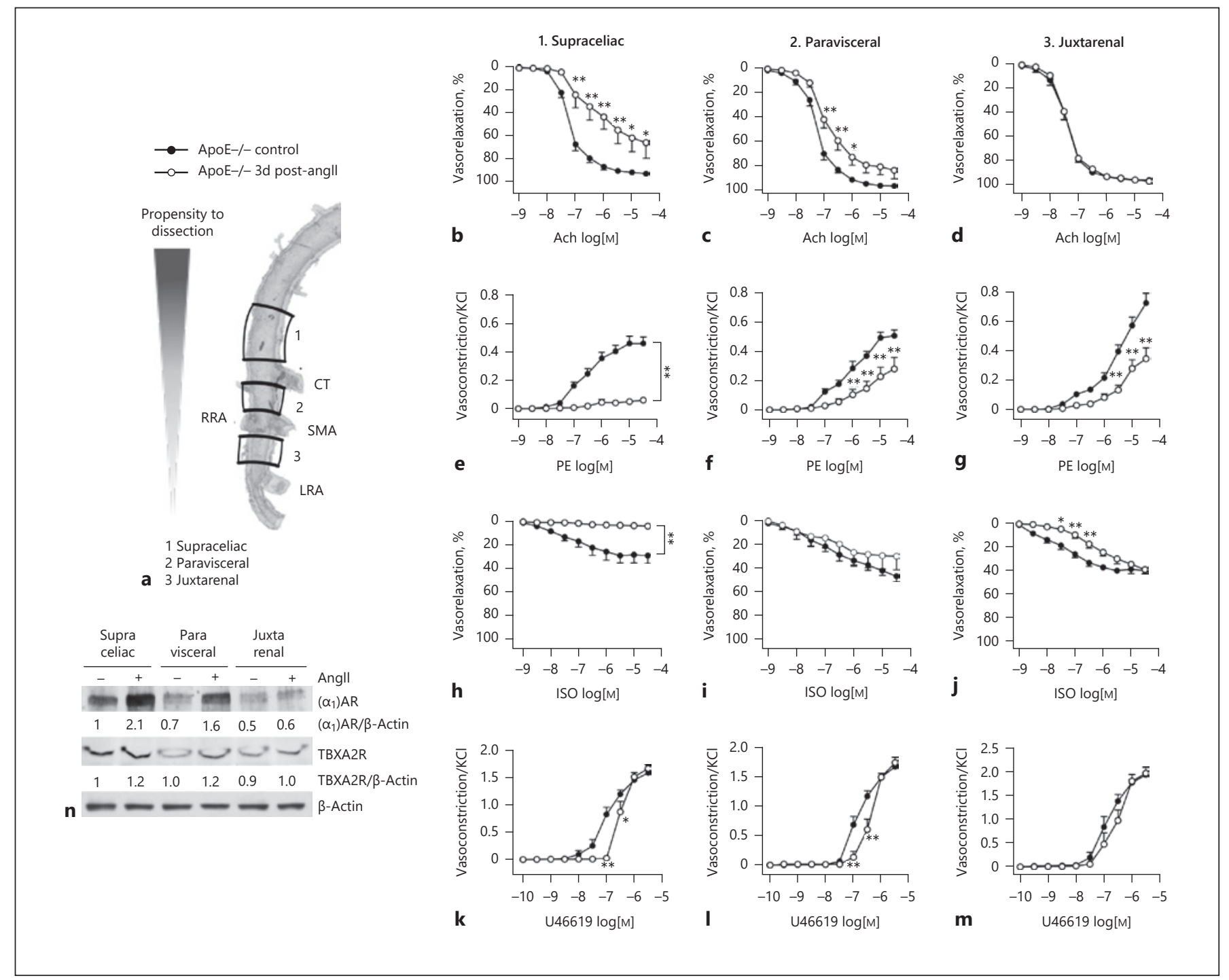

Fig. 2. Vascular functions of the supraceliac, paravisceral, and juxtarenal aortic segments. a Schematic representation of the (1) supraceliac, (2) paravisceral, and (3) juxtarenal aortic segments evaluated for vascular reactivity in the wire myograph system. b-d Concentration-response curve to $\mathrm{ACH}$-induced vasodilation of vascular segments pre-contracted with serotonin $\left[10^{-6} \mathrm{M}\right]$. PE $(\mathbf{e - g})$, ISO (h-j) and U46619 (k-m), TXA2 analog, concentrationresponse curve normalized to $\mathrm{KCl}(60 \mathrm{mM})$-induced vasoconstric-

\section{Western Blot Analysis of Aortic Segments}

Cleaned from connective and fat tissue, supraceliac, paravisceral, and juxtarenal segments were stored at $-80^{\circ} \mathrm{C}$. For WB analysis, 5-6 segments per group were pulled, homogenized in RIPA buffer, and lysates were analyzed with sodium dodecyl sulfatepolyacrylamide gel electrophoresis (SDS-PAGE) and immunoblotting. The following primary antibodies were used for WB analysis: $\alpha_{1 A}$-AR (\#sc-100291, SCBT, TA, USA), TBXA2R (\#PA567751, Thermo Scientific, Waltham, MA, USA), and $\beta$-actin (\#A2228, Sigma, St. Louis, MO, USA). tions. Data are expressed as the mean \pm SEM. Statistical significance was determined by two-way ANOVA with Bonferroni's multiple comparisons post hoc test. ${ }^{*} p \leq 0.05 ;{ }^{* *} p \leq 0.01, n=5-7$ per group. $\mathbf{n}$ Western blot and densitometric analysis ofAR and TBXA2R expression in supraceliac, paravisceral, and juxtarenal aortic segments; $\beta$-Actin, loading control, $n=5-6$ segments per group.

\section{Statistics}

Changes in micro-CT aortic volume between baseline and end point geometry were evaluated with ordinary one-way ANOVA with Dunnett's multiple comparisons test. Changes in contrast agent volume infiltration between time points were assessed using Kruskal-Wallis tests with Dunn's multiple comparisons. For the vascular reactivity studies, statistical significance was determined using two-way ANOVA with Bonferroni's multiple comparisons post hoc test. Statistical significance was indicated by $p \leq 0.05$. 
Table 1. EC50 and $E_{\max }$ values of U46619-induced vasoconstriction in different abdominal segments

\begin{tabular}{|c|c|c|c|c|c|c|}
\hline \multirow[t]{2}{*}{ Fitted parameter } & \multicolumn{2}{|c|}{ Supraceliac } & \multicolumn{2}{|c|}{ Paravisceral } & \multicolumn{2}{|c|}{ Juxtarenal } \\
\hline & controls & AngII & controls & AngII & controls & AngII \\
\hline $\begin{array}{l}\mathrm{EC} 50,10^{-7} \mathrm{M} \\
p \text { value }\end{array}$ & $\begin{array}{c}1.190 \\
<0.0001\end{array}$ & 5.245 & $\begin{array}{c}1.941 \\
<0.0001\end{array}$ & 7.191 & $\begin{array}{l}1.662 \\
0.0021\end{array}$ & 3.895 \\
\hline $\begin{array}{l}E_{\max } \\
p \text { value }\end{array}$ & $\begin{array}{l}1.654 \\
0.0006\end{array}$ & 2.072 & $\begin{array}{l}1.799 \\
0.0009\end{array}$ & 2.241 & $\begin{array}{l}2.062 \\
0.1489\end{array}$ & 2.306 \\
\hline
\end{tabular}

Values of EC50 and $E_{\max }$ were compared using extra-sum-of-squares $F$ test.

\section{Results}

Longitudinal Assessment of AngII-Induced Changes in Aortic Volume and Microstructure

In order to identify the initial vascular changes from the later remodeling, vascular segments prone to AA and $\mathrm{AD}$ were assessed at 1,2 , and 3 days post-AngII infusion for volumetric and structural damages. Micro-CT measurements showed a significant increase in the volume of the abdominal aorta of ApoE-/- mice at 3 days post-AngII infusion (Fig. 1a, b). The volumetric enlargement of the abdominal aorta in vivo was paralleled by intramural infiltration of the contrast agent (Fig. 1c-e; online suppl. Fig. 1; see www.karger.com/doi/10.1159/000509545 for all online suppl. material), indicating early vascular damage [22]. The intravascular leak of the contrast agent was significant at day 3 post-AngII infusion. Interestingly, although this observation was consistent for all 3 investigated suprarenal regions, the amount of infiltrated contrast agent was higher in the supraceliac and paravisceral segments than in the juxtarenal segment (Fig. 1c-e).

\section{Spatial Characterization of Endothelial and SMC}

Function of Aortic Segments Prone- and Resistant to AA/AD at 3 Days Post-AngII

To understand whether the increase in aortic volume and micro-damage at 3 days post-AngII was paralleled by functional impairments, the vascular reactivity of the supraceliac, paravisceral, and juxtarenal aortic segments, as well as of 3 aortic side branches, was evaluated using the wire myograph system. We observed spatial-selective degrees of endothelial dysfunction in the 3 abdominal aortic segments (Fig. 2b-d). Endothelium-dependent vasorelaxation to acetylcholine was dramatically diminished in the supraceliac and the paravisceral regions, whereas it was completely preserved in most distal juxtarenal segment compared to ApoE-/- controls.

Early Morphofunctional Changes in Aortopathologies
The function of SMCs was also differentially impaired along the AngII-infused aorta. PE-induced vasoconstriction, to an al-adrenoreceptor agonist, was blunted in the supraceliac segment, whereas it was present in both the paravisceral and juxtarenal segments, albeit with a significant reduction (Fig. 2e-g). The relaxation in response to isoproterenol, a nonselective $\beta$-receptor agonist, was also significantly decreased in the supraceliac segment (Fig. $2 \mathrm{~h}-\mathrm{j}$ ). We additionally assessed the vasoconstriction induced by U46619, a stable thromboxane A2 (TXA2) mimetic. While the maximal constriction induced by U46619 was not different among the 3 vascular segments (Fig. $2 \mathrm{k}-\mathrm{m}$ ), there was a significant right shift of the concentration-response curve in the supraceliac and paravisceral, but not the juxtarenal segments (Table 1). To assess whether the reduced contraction to $\mathrm{PE}$ and U46619 was due to the downregulation of their receptors, WB analysis was performed on these vascular segments. Surprisingly, $\left(\alpha_{1}\right)$ AR expression was upregulated in all 3 aortic segments (Fig. $2 \mathrm{n}$ ), whereas TBXA2R was no different. The vasoconstriction data have been normalized to $\mathrm{KCl}$-induced vasoconstrictions (online suppl. Fig. 2).

Spatial Characterization of Endothelial and SMC

Functions of Aortic Side Branches

In addition to the suprarenal aorta segments, we also assessed the vasoreactivity of the 3 abdominal aortic side branches, including the celiac, superior mesenteric, and left renal segment (Fig. 3a). Endothelium-mediated vasorelaxation to $\mathrm{ACH}$ was preserved in all the branches, with the exception of the left renal artery (Fig. 3b-d). Vasoconstriction in response to $\mathrm{PE}$ and TXA2 analog was retained in all the side branches (Fig. $3 \mathrm{e}-\mathrm{j}$ ). These findings demonstrate that the vascular function of the side branches was overall preserved, in agreement with the dissectionresistant features of these vascular regions. 


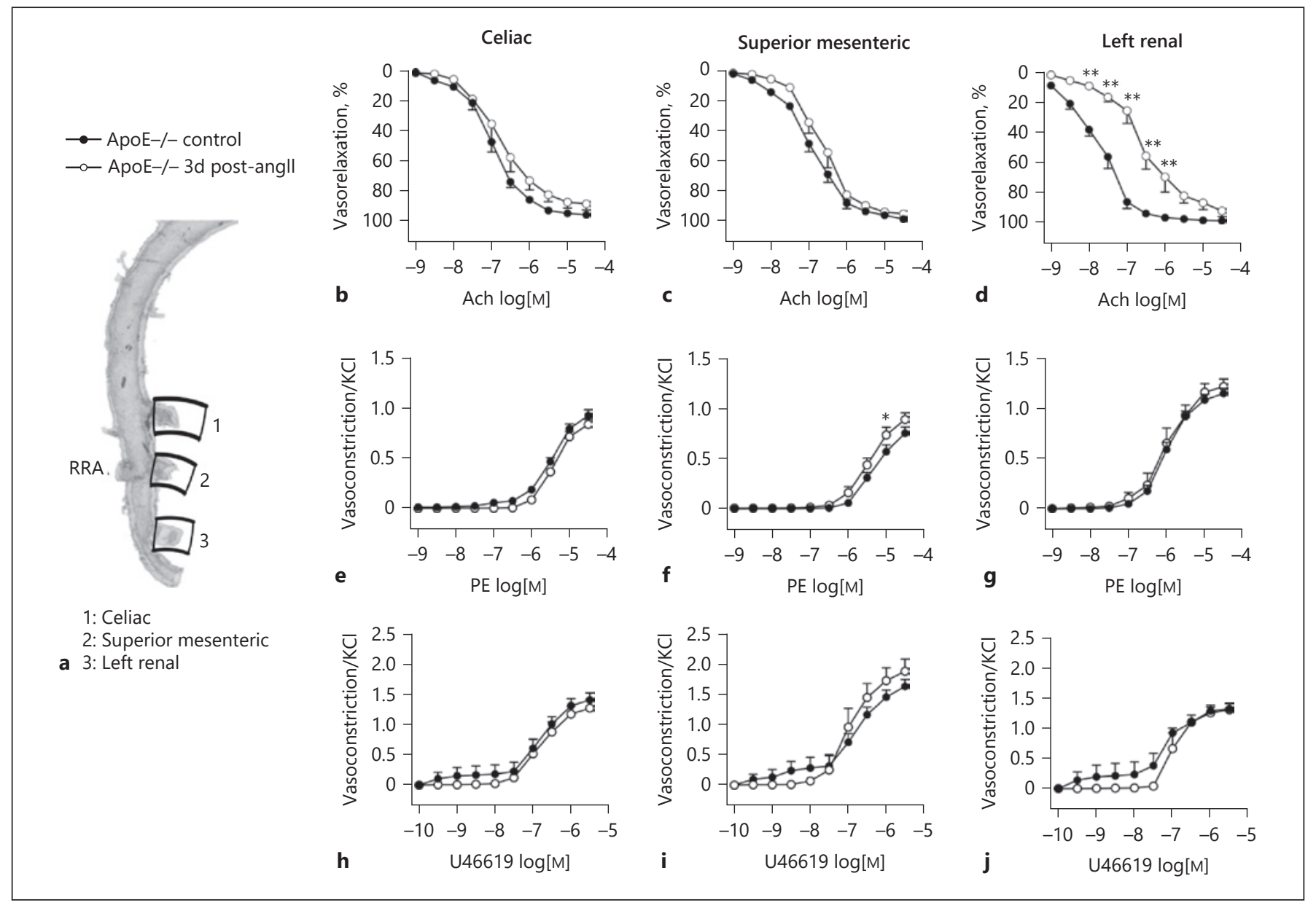

Fig. 3. Vascular functions of the celiac, superior mesenteric, and left renal segment. a Schematic representation of the (1) celiac, (2) superior mesenteric, and (3) left renal aortic segments evaluated for vascular reactivity by using the wire myograph system. b-d Concentration-response curve to Ach-induced vasodilation of vascular segments pre-contracted with serotonin $\left[10^{-6} \mathrm{M}\right]$. PE

\section{Discussion}

Numerous studies have been published on the pathogenesis of ascending [27, 28] and abdominal lesions [18] using the AngII infusion mouse model of AA and AD. However, pathological mechanisms dictating the spatial and longitudinal development of the diseases remain poorly understood. During the first 3 days of infusion, AngII induces a raise of mean blood pressure of ca. 20 $\mathrm{mm} \mathrm{Hg}$, followed by further increase [29]. Saraff et al. [10] reported that at day 2 post-AngII infusion, the fragmentation of elastin correlates with macrophage infiltration in the media of the suprarenal aorta of ApoE-/mice. We reported the presence of microruptures at day (e-g), U46619 (h-j), TXA2 analog, concentration-response curve normalized to $\mathrm{KCl}(60 \mathrm{mM})$-induced vasoconstrictions. Data are expressed as mean \pm SEM. Statistical significance was determined by two-way ANOVA with Bonferroni's multiple comparisons post hoc test. ${ }^{*} p \leq 0.05 ;{ }^{* *} p \leq 0.01, n=5-7$ per group.

3 post-AngII infusion, which was supported by the increase of MMP-2 and MMP-13 expression in the suprarenal and ascending aorta at day 4 postimplantation reported by Bersi et al. [18]. These findings suggest the establishment of an active inflammatory and remodeling process as early as day 4 following AngII infusion.

Here, by using synchrotron imaging, we detected a significant intramural infiltration of contrast agent, index of vascular damage [22], as early as day 3 post-AngII infusion, in the abdominal aorta of ApoE-/- mice (Fig. 1; online suppl. Fig. 1). Similarly, at day 3 postimplantation, a significant increase in the abdominal aorta volumes was measured by contrast-enhanced micro-CT. In spite of the systemic administration of AngII, AD are almost exclusively localized 
in the suprarenal abdominal aorta $[8,10,11]$. In a previous study, we demonstrated a co-localization of excessive strain around the celiac and superior mesenteric branches of the abdominal aorta with microstructural wall damage at day 3 post-AngII infusion, suggesting a mechano-driven initiation of the disease [22]. Bersi et al. [18] recently suggested that insufficient collagen deposition in the suprarenal aorta may render the region vulnerable to wall stress and, hence, to dissection. Our data revealed an early onset of regional vascular dysfunction, which may contribute to the impaired stress shielding.

Interestingly, among the 3 suprarenal aortic segments, the supraceliac region presents the most profound endothelial dysfunction (Fig. 2e), which is associated with a significant intramural contrast agent infiltration, index of micro-damage in the vascular wall (Fig. 1). Previous studies have reported endothelial dysfunction of different aortic regions but at much later time points. For instance, endothelial impairment of the thoracic aorta and femoral artery has been reported at day 14 and 28 post-AngII infusion in ApoE-/- mice [23, 30]. Our findings demonstrate a regional endothelial dysfunction preceding the onset of dissection. At 3 days post-AngII infusion, whereas endothelial function was blunted in the supraceliac aortic segment, it was preserved in the celiac and superior mesenteric side branches, and partially retained in the left renal artery (Fig. 3b-d).

Biomechanical studies have shown that the area around the orifice of side branches is subject to focal strain in the early stage of AngII infusion [22], particularly the ostia of the celiac and superior mesenteric arteries. Following AngII treatment, BP markedly increases [29], imposing a significant mechanical stress on the vascular wall. In response to high pressure, the vascular wall contracts to counterbalance the increase in wall tension through a reduction in diameter, following Laplace's law. Interestingly, the vasoconstriction in response to PE and $\mathrm{TXA}_{2}$ mimetic were markedly suppressed in the supraceliac segment (Fig. 2e, $\mathrm{k}$ ), which is also the most dissectionprone region within the suprarenal aorta [31]. Furthermore, the vasoconstriction was regionally compromised and followed a tapering trend toward the distal end of the aorta, with the side branches fully preserving their vasoconstrictive functions. Studies have reported that the C57BL/6 thoracic aorta showed no change in PE-induced contractions after 21 days of infusion [32]; however, those findings focused on later time points. Interestingly, subpressor AngII doses in rats increased both $\mathrm{PE}$ and $\mathrm{KCl}$ contractions in the aorta $[33,34]$. However, it is difficult to reconcile vascular reactivity data performed with dif- ferent conditions of time points, doses of AngII infusion, and animal species $[35,36]$. In contrast to the blunted vasoconstriction in response to $\mathrm{PE}, \mathrm{WB}$ analysis (Fig. 2n) revealed an upregulation of $\alpha 1$ (AR) in these aortic segments, suggesting that a downregulation of the receptor was not accountable for the observed phenotype. It is possible that receptor activation and/or signal transduction are impaired. Additional studies are needed to dissect the mechanisms underlying this effect.

Our results indicate an early and spatially confined suppression of a1-adrenergic receptor-mediated contractility of suprarenal aortic segment in the AngII-infused ApoE-/- mice, which implies an impaired capacity of this vascular region to counterbalance the pressureinduced mechanical stress. The exposure of the wall to high loads likely contributes to the site-specific onset of dissection.

In conclusion, our study is the first to demonstrate the regional onset of endothelial and smooth muscle dysfunctions in the suprarenal aortic segment at 3 days postAngII infusion, compared to other neighboring aortic regions and branches. Furthermore, these localized vascular dysfunctions tightly correlate with microstructural damages and in vivo increase of the aortic volume. The focal transition of endothelial and contractile properties from dysfunctional (aorta) to functional (branches) might further contribute to the mechanical burden that branch orifices endure and may, therefore, be an important factor in the onset of dissections in mice. Our findings pave the way for further studies on the molecular mechanisms dictating the regional loss of endothelial and contractile functions.

\section{Acknowledgement}

The authors thank the EPFL Histology Facility for staining the histological sections and acknowledge the Paul Scherrer Institute, Villigen, Switzerland for provision of synchrotron radiation beamtime at beamline X02DA TOMCAT of the SLS.

\section{Statement of Ethics}

Animal experiments conform to internationally accepted standards and have been approved by the appropriate institutional review body.

\section{Conflict of Interest Statement}

The authors have no conflicts of interest to declare. 


\section{Funding Sources}

This work was supported, in part, by the Swiss National Science Foundation (SNF) grant CR23I2_163370 (N.S.), Research Foundation-Flanders (FWO) fellowship 12A5816N, and Research Foundation-Flanders (FWO) project G086917N (B.T.). This work was also supported by the National Heart, Lung, and Blood Institute of the National Institutes of Health grant R01 HL126913 (A.D.L.) and National Institute of Neurological Disorders and Stroke of National Institutes of Health grant R21 NS104512 (A.D.L.).

\section{Author Contributions}

L.A., B.T., A.D.L., and N.S. were involved in the experiment design. L.A., B.T., G.L., and L.S. performed the experiments. L.A., B.T., and A.D.L. analyzed the data. L.A., B.T., and A.D.L. wrote the manuscript. G.L. edited the manuscript. All authors read and approved the final manuscript.

\section{References}

1 Deaths, percent of total deaths, and death rates for the 15 leading causes of death in 5 -year age groups, by race, and sex: United States, 2015 [Internet]. 2015. Available from: https: //www.cdc.gov/nchs/data/dvs/ lcwk1_2015.pdf.

2 Atkins MD, Black JH, Cambria RP. Aortic dissection: perspectives in the era of stentgraft repair. J Vasc Surg. 2006;43(Suppl A): 30A-43A.

3 Crawford TC, Beaulieu RJ, Ehlert BA, Ratchford EV, Black JH 3rd. Malperfusion syndromes in aortic dissections. Vasc Med. 2016; 21(3):264-73.

4 Singh M, Ziganshin BA, Elefteriades JA. Aortic aneurysm. In: Vasan RS, Sawyer DB, editors. Encyclopedia of cardiovascular research and medicine. Oxford: Elsevier; 2018. p. 12342.

5 Quintana RA, Taylor WR. Cellular mechanisms of aortic aneurysm formation. Circ Res. 2019;124(4):607-18.

$6 \mathrm{Lu} \mathrm{H}$, Rateri DL, Bruemmer D, Cassis LA, Daugherty A. Involvement of the renin-angiotensin system in abdominal and thoracic aortic aneurysms. Clin Sci. 2012;123(9):53143.

7 Luo F, Zhou XL, Li JJ, Hui RT. Inflammatory response is associated with aortic dissection. Ageing Res Rev. 2009;8(1):31-5.

8 Daugherty A, Manning MW, Cassis LA. Angiotensin II promotes atherosclerotic lesions and aneurysms in apolipoprotein E-deficient mice. J Clin Invest. 2000;105(11):1605-12.

9 Daugherty A, Rateri DL, Charo IF, Owens AP, Howatt DA, Cassis LA. Angiotensin II infusion promotes ascending aortic aneurysms: attenuation by CCR2 deficiency in apoE-/mice. Clin Sci. 2010;118(11):681-9.

10 Saraff K, Babamusta F, Cassis LA, Daugherty A. Aortic dissection precedes formation of aneurysms and atherosclerosis in angiotensin II-infused, apolipoprotein E-deficient mice. Arterioscler Thromb Vasc Biol. 2003 Sep 1; 23(9):1621-6.

11 Phillips EH, Di Achille P, Bersi MR, Humphrey JD, Goergen CJ. Multi-modality imaging enables detailed hemodynamic simulations in dissecting aneurysms in mice. IEEE Trans Med Imaging. 2017;36(6):1297-305.

12 Swafford AN, Harrison-Bernard LM, Dick GM. Knockout mice reveal that the angioten- sin II type $1 \mathrm{~B}$ receptor links to smooth muscle contraction. Am J Hypertens. 2007;20(3): 335-7.

13 Zhou Y, Chen Y, Dirksen WP, Morris M, Periasamy $M$. AT1b receptor predominantly mediates contractions in major mouse blood vessels. Circ Res. 2003;93(11):1089-94.

14 Zhou Y, Dirksen WP, Babu GJ, Periasamy M. Differential vasoconstrictions induced by angiotensin II: role of $\mathrm{AT} 1$ and $\mathrm{AT} 2$ receptors in isolated C57BL/6J mouse blood vessels. Am J Physiol Heart Circ Physiol. 2003;285(6): H2797-803.

15 Masi S, Uliana M, Virdis A. Angiotensin II and vascular damage in hypertension: Role of oxidative stress and sympathetic activation. Vascul Pharmacol. 2019;115:13-7.

$16 \mathrm{Ju}$ X, Tilton RG, Brasier AR. Multifaceted role of angiotensin II in vascular inflammation and aortic aneurysmal disease. In: Grundma$\mathrm{nn} \mathrm{R}$, editor. Etiology, pathogenesis and pathophysiology of aortic aneurysms and aneurysm rupture. Rijeka: IntechOpen; 2011. p. 119-36.

17 Forrester SJ, Booz GW, Sigmund CD, Coffman TM, Kawai T, Rizzo V, et al. Angiotensin II signal transduction: an update on mechanisms of physiology and pathophysiology. Physiol Rev. 2018;98(3):1627-738.

18 Bersi MR, Khosravi R, Wujciak AJ, Harrison DG, Humphrey JD. Differential cell-matrix mechanoadaptations and inflammation drive regional propensities to aortic fibrosis, aneurysm or dissection in hypertension. J R Soc Interface. 2017; 14(136):20170327.

19 Trachet B, Aslanidou L, Piersigilli A, FragaSilva RA, Sordet-Dessimoz J, Villanueva-Perez $\mathrm{P}$, et al. Angiotensin II infusion into ApoE-/- mice: a model for aortic dissection rather than abdominal aortic aneurysm? Cardiovasc Res. 2017;113(10):1230-42.

20 Trachet B, Fraga-Silva R, Piersigilli A, Tedgui A, Sordet-Dessimoz J, Astolfo A, et al. Dissecting abdominal aortic aneurysm in ang IIinfused mice: suprarenal branch ruptures and apparent luminal dilatation. Cardiovasc Res. 2015;105(2):213-22.

21 Trachet B, Lovric G, Villanueva-Perez P, Aslanidou L, Ferraro M, Logghe G, et al. Synchrotron-based phase contrast imaging of cardiovascular tissue in mice-grating interfer- ometry or phase propagation? Biomed Phys Eng Express. 2018;5(1):015010.

22 Aslanidou L, Ferraro M, Lovric G, Bersi MR, Humphrey JD, Segers P, et al. Co-localization of microstructural damage and excessive mechanical strain at aortic branches in angiotensin-II-infused mice. Biomech Model Mechanobiology. 2020;19(1):81-97.

23 Fransen P, Van Hove CE, Leloup AJ, Schrijvers DM, De Meyer GR, De Keulenaer GW. Effect of angiotensin II-induced arterial hypertension on the voltage-dependent contractions of mouse arteries. Pflugers Arch. 2016 Feb;468(2):257-67.

24 Korneva A, Humphrey JD. Maladaptive aortic remodeling in hypertension associates with dysfunctional smooth muscle contractility. Am J Physiol Heart Circ Physiol. 2019; 316(2):H265-78.

25 Ferruzzi J, Murtada SI, Li G, Jiao Y, Uman S, Ting MY, et al. Pharmacologically improved contractility protects against aortic dissection in mice with disrupted transforming growth factor- $\beta$ signaling despite compromised extracellular matrix properties. Arterioscler Thromb Vasc Biol. 2016;36(5):91927.

26 Logghe G, Trachet B, Aslanidou L, Villaneuva-Perez P, De Backer J, Stergiopulos N, et al. Propagation-based phase-contrast synchrotron imaging of aortic dissection in mice: from individual elastic lamella to 3D analysis. Sci Rep. 2018;8(1):2223.

27 Trachet B, Piersigilli A, Fraga-Silva RA, Aslanidou L, Sordet-Dessimoz J, Astolfo A, et al. Ascending aortic aneurysm in angiotensin II-infused mice: formation, progression, and the role of focal dissections. Arterioscler Thromb Vasc Biol. 2016;36(4):67381.

28 Rateri DL, Davis FM, Balakrishnan A, Howatt DA, Moorleghen JJ, O'Connor WN, et al. Angiotensin II induces region-specific medial disruption during evolution of ascending aortic aneurysms. Am J Pathol. 2014;184(9): 2586-95.

29 Haggerty CM, Mattingly AC, Gong MC, Su W, Daugherty A, Fornwalt BK. Telemetric blood pressure assessment in angiotensin IIinfused ApoE-/- mice: 28 day natural history and comparison to tail-cuff measurements. PloS One. 2015;10(6):e0130723. 
30 Seto SW, Krishna SM, Yu H, Liu D, Khosla S, Golledge J. Impaired acetylcholine-induced endothelium-dependent aortic relaxation by caveolin-1 in angiotensin II-infused apolipoprotein-E (ApoE-/-) knockout mice. PloS One. 2013;8(3):e58481.

31 Favreau JT, Nguyen BT, Gao I, Yu P, Tao M, Schneiderman J, et al. Murine ultrasound imaging for circumferential strain analyses in the angiotensin II abdominal aortic aneurysm model. J Vasc Surg. 2012;56(2):462-9.

32 Zhou Z, Yadav VR, Sun C, Teng B, Mustafa JS. Impaired aortic contractility to uridine ad- enosine tetraphosphate in angiotensin II-induced hypertensive mice: receptor desensitization? Am J Hypertens. 2017;30(3):304-12.

33 Gallardo-Ortíz IA, Rodríguez-Hernández SN, López-Guerrero JJ, Del Valle-Mondragón L, López-Sánchez P, Touyz RM, et al. Role of a1D-adrenoceptors in vascular wall hypertrophy during angiotensin II-induced hypertension. Auton Autacoid Pharmacol. 2015;35(3): 17-31.

34 Bao W, Behm DJ, Nerurkar SS, Ao Z, Bentley R, Mirabile RC, et al. Effects of p38 MAPK inhibitor on angiotensin II-dependent hyper- tension, organ damage, and superoxide anion production. J Cardiovasc Pharmacol. 2007; 49(6):362-8.

35 Cassis LA, Huang J, Gong MC, Daugherty A. Role of metabolism and receptor responsiveness in the attenuated responses to angiotensin II in mice compared to rats. Regul Pept. 2004;117(2):107-16.

36 Phillips EH, Chang MS, Gorman S, Qureshi HJ, Ejendal KFK, Kinzer-Ursem TL, et al. Angiotensin II infusion does not cause abdominal aortic aneurysms in apolipoprotein E-deficient rats. J Vasc Res. 2018;55(1):1-12. 